\title{
Design of the Intelligent Air Humidifier
}

\author{
Biqing Li ${ }^{1,}$ a , Wenya Lai ${ }^{1, a, ~}{ }^{*}$, Chongjun Yang ${ }^{1,}$ a , Shiyong Zheng', b \\ ${ }^{1}$ College of Mechanical and Electronic Engineering, Hezhou University, Hezhou Guangxi 542899, \\ China \\ ${ }^{2}$ College of Computer Science and Information Engineering, Hezhou University, Hezhou Guangxi \\ 542899, China \\ ajanliful@163.com, b229292710@qq.com
}

Keywords: SCM, intelligence, humidifier, relative moisture, sensor.

\begin{abstract}
The ordinary sensors require manual control and do not have the indoor air humidifier test function. This design mainly adopts STC89C52RC SCM control, and connects with the auxiliary circuit to achieve automatic test and sound-light alarm of the intelligent air humidifier. The intelligent air humidifier can start, close and test indoor temperature and moisture automatically. It is small, easy to carry and has certain practical value.
\end{abstract}

\section{Hardware design}

The system consists of the master control circuit, the temperature and moisture acquisition circuit, the buzzer, the LDE light (sound-light alarm) alarm circuit and LCD display circuit. The master control circuit conducts comprehensive processing of the data collected through the external I/O interface, and the processed data are displayed by LCD. At the same time, the processed data are compared with the preset data. Based on the comparison results, the operation of the relay circuit, the buzzer alarm circuit and the motor-driven circuit are controlled so as to achieve sound-light alarm and the automatic humidification effect. The system's overall design framework is shown in Figure 1.

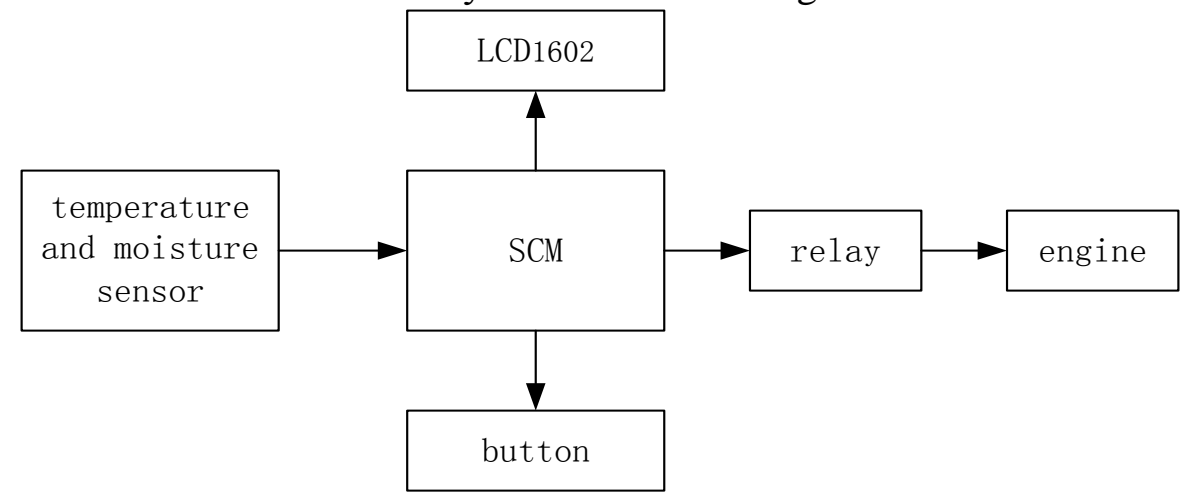

Fig. 1 Overall design block diagram

The master control part adopts the minimal system of the STC89C52RC SCM. The minimal system includes the SCM and its accessories, including external power source, clock circuit, and manual reset circuit. It can guarantee the SCM at a normal operation status. The function of the clock circuit is to provide a clock period for the master circuit.

The temperature and humidity acquisition circuit consists of the AM2302 temperature and humidity sensor and accessories. It is mainly responsible for testing temperature and humidifier. When the SCM emits the test signal, the sensor will make some response to collect data. The collected data is then sent to the SCM. After the SCM analyzes and processes the data, the SCM outputs control signals. The control display can display the value of temperature and humidifier tested in the form of numerical signals.

The LCD1602 display circuit chooses LCD1602 as its displaying part. The displayer can show the tested and preset temperature and humidity, and other real-time information. 
In this design, the piezoelectric active buzzer is adopted as the auditory cue part of the system; the indicator light is adopted as the optical reminder. The SCM collects humidity data (namely the value of humidity of the current air) with the preset value. If the former is lower than the latter, the SCM can send out control signals to make the buzzer send out sound alarm and make the indicator light sparkle to alarm. In other words, when the indoor humidity is lower than the present value, the humidifier give out an alarm and the indicator light will spark to alarm to remind the owner that the current indoor air humidity is lower than the present value and that the humidifier has been automatically launched for humidification. When the air humidity reaches the preset value, the buzzer will automatically stop working, so will the indicator light.

\section{Software design}

After the system is launched, the LCD is first initialized and the program is loaded in to read the preset value of the timer. Then, temperature and humidity data collection, real-time time reading, button scanning and engine scanning are conducted in turn. The program will finally calculate the data collected from the program flow and the preset data, and compare them. The system will work out the comparison results. According to the comparison results, corresponding control instructions are sent to various functional modules. Every module makes corresponding movement according to the corresponding task to realize its functions and functions required of the whole design. The step is repeated until the start of the next round of data collection.

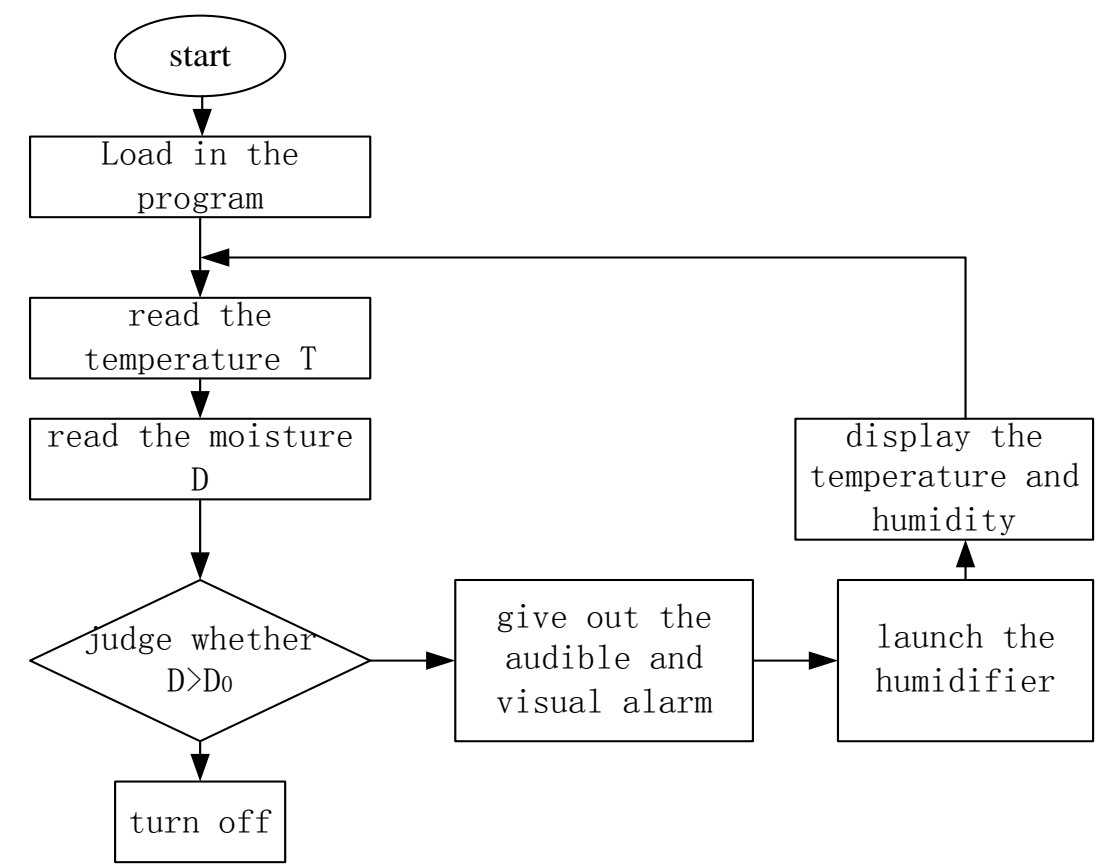

Fig. 2 Total system program flowcharts

After initialization is started, the program is loaded in. According the displayed data, the temperature (T) and the humidity (D) can be read. Then, compare the humidity (D) and the set value (D0). If $\mathrm{D}<\mathrm{D} 0$, the audible and visual alarm and the humidifier are launched to conduct air humidification and display temperature and humidity. The alarm will keep on until the air humidity is $\mathrm{D}<=$ Do. In other words, the humidifier humidifies the air to achieve the desire humidity. At the moment, the humidifier will be automatically turned off and the temperature and humidity process circle will be displayed. 


\section{Conclusion}

The intelligent gadgets designed based on the SCM has achieved better development for the increasingly intelligent society. As a core control item, the SCM has achieved bright application prospects in various control fields. The intelligent humidifier designed in this paper can preset the value of humidity according to the practical situations. When the humidification degree reaches the preset value, the humidifier will stop working automatically, but will keep on detecting the air humidifier. Once the humidity decreases to the preset value, it will keep on humidifying so as to achieve the function of intelligent humidification and avoid resulting in an excessively high humidity.

\section{Acknowledgements}

This work is supported the following fund:

2016 The project of improving the basic ability of young teachers in Colleges and universities in Guangxi: "Design and development of electronic commerce platform of agricultural products based on Semantic Technology"(No,KY2016YB455).

2016 Guangxi higher education teaching reform project: "Exploration on the cultivation mechanism of the teaching characteristics of Communication Engineering Specialty Based on ZTE ICT education platform".

2015 college students' innovative training program: "Research on the application of value added travel experience in the mobile terminal of the "ethnic custom travel' in Guangxi" (No 201511838070); \&\& "The design and development HeYuanTong Campus Mobile Phone APP based on Android" (No 201511838034).

2015 Teaching case project construction project of hezhou university: "Tourist positioning and guiding system project of Huangyao scenic"; \&\& "Development and application of the information management system of 'Huang Yao Tong'".

Project of scientific research and technology development project of Hezhou: "Design and implementation of agricultural products e-commerce platform based on Semantic Technology" (No, Hekeneng 1506006)

Scientific research project of hezhou university: "Research and development of E-government platform based on mobile terminal" (No 2015ZZZK03);

Reform in Education project of hezhou university: "Internet of things" (No hzxytszy201501); "Research on application personnel training model of Communications for SMEs" (No hzxyjg201525);

Master degree discipline construction scientific research and Cultivation Project: "Research on the social work service of the teenagers' Network Addiction" (No 2015SHGZ005) \&\&"Research on the intelligent development of rural tourism in Guangxi based on the Internet of things" (No 2015MTA16).

\section{References}

[1] LI Guangdi, ZHU Yuexiu \& LENG Zuqi. SCM Basic [M]. 3rd Edition, Beihang University Press, 2011.

[2] ZHU Chenggao. Manuals for Electricians and Electronic Techniques[M]. Beijing: Higher Education Press, 2012.

[3] LI Wenzhen. Simplified timer based on STC89C52 [J]. Science and Technology, 2014 (3): 67-68.

[4] ZHANG Yigang. Fundamentals of SCM and Interface Techniques [M]. Beijing: Posts and Telecom Press, 2011. 